\title{
APPLICATION OF NUCLEATION THEORY TO THE PROCESS OF DROPLET FREEZING ON AN ICE SUBSTRATE
}

\author{
By L. Levi \\ (Física del Sólido, Departmento de Investigaciónes, Comisión Nacional de Energía Atómica, \\ Buenos Aires, Argentina)
}

Abstract. The probability $P$ that ice embryos, nucleated inside droplets colliding with an ice surface, have crystallographic orientations different from that of the substrate, is correlated with the mean number $\bar{N}$ of droplets which form each crystal in accretions grown in dry regime (substrate temperature $T_{\mathrm{s}}<\mathrm{o}^{\circ} \mathrm{C}$ ). The theory of heterogeneous nucleation is applied to represent the ice embryo nucleation rate and the approximate expression $P \approx \mathrm{I} / \bar{N} \approx P_{0} \exp \left(-\Delta G_{\gamma} \star / k T\right)$ is obtained, where $\Delta G_{\gamma}{ }^{\star}$ is the nucleation energy for randomly oriented critical embryos. Assuming cap-shaped embryos and taking into account the crystal size in accretions grown at $T_{\mathrm{s}}=-\mathrm{I} 5^{\circ} \mathrm{C}$ and air temperatures $-\mathrm{I} 8^{\circ} \mathrm{C} \geqslant T_{\mathrm{a}} \geqslant$ $\geqslant-27^{\circ} \mathrm{C}$, it is found that $P_{0}=\mathrm{I} 25^{\circ}, \Delta G_{\gamma}{ }^{\star}=\mathrm{I} .5 \times 10^{-10} / \Delta T^{2} \mathrm{erg}$.

The relationship between the value of $\Delta G_{\gamma}{ }^{\star}$ and those of the crystal-embryo and crystalmelt interfacial free energies is discussed. Finally the dependence of $P_{0}$ on the growth conditions and on the ice substrate orientation is analysed, considering the effect of embryo breeding by collision and that of orientation selection, which is responsible for the establishment of preferential orientations in the accretions. 